Chinese Journal of Organic Chemistry

\title{
氟嗍二异吲哚类化合物的合成及光学性能表征
}

\begin{tabular}{|c|c|c|}
\hline 杨晓霞 $a, b$ & 淮 茾 ${ }^{b}$ & 蔡良珍 $*, b$ \\
\hline & 大学化学化工与生物工程学院 & 上海 201620) \\
\hline & ${ }^{b}$ 华东理工大学化学与分子工程学院 & 上海 200237) \\
\hline
\end{tabular}

\begin{abstract}
摘要 以取代的苯甲酸乙酯和邻羟基苯乙酮为原料, 合成了一系列含卤素取代氟硼二异吲哚类新化合物. 这些化合物 的结构均通过了 ${ }^{1} \mathrm{H}$ NMR, HRMS, IR 等手段的表征. 通过紫外可见吸收光谱和荧光发射光谱测试, 化合物均显示较长 的紫外吸收及苂光发射波长. 取代基对于化合物摩尔消光系数和荧光量子产率以及单线态氧生成能力有明显的影响, 其中含 $\mathrm{CH}_{3} \mathrm{O}$ 和 $\mathrm{CF}_{3}$ 取代的化合物 $\mathbf{6 b}$ 和 $\mathbf{6 f}$ 显示了更高的摩尔消光系数, 而含 $\mathrm{Br}$ 取代的化合物 $\mathbf{6 e}$ 显示高的单线态氧 生成能力和中等的苂光量子产率.
\end{abstract}

关键词 氟嗍二异吲哚; 合成; 荧光; 紫外; 单线态氧

\section{Synthesis of Boron Diisoindolmethene Compounds and Spectral Properties}

\author{
Yang, Xiaoxia ${ }^{a, b} \quad$ Lin, Zhixing $^{b} \quad$ Lin, Hui $^{b} \quad$ Cai, Liangzhen ${ }^{*, b} \quad$ Tao, Xiaochun ${ }^{*, b}$ \\ ( ${ }^{a}$ College of Chemistry, Chemical Engineering and Biotechnology, Donghua University, Shanghai 201620 ) \\ ( ${ }^{b}$ School of Chemistry and Molecular Engineering, East China University of Science and Technology, Shanghai 200237)
}

\begin{abstract}
The new boron diisoindolmethene compounds were synthesized from ethyl benzoate and 1-phenylethanone, and characterized by ${ }^{1} \mathrm{H}$ NMR, HRMS and IR. These compounds exhibited the long UV-absorption and fluorescence emission wavelength. The effects of substituents on the molar extinction coefficient, fluorescence quantum yield and singlet oxygen yield were easily noticed, methoxy substituted compound $\mathbf{6 b}$ and $\mathrm{CF}_{3}$ substituted compound $\mathbf{6} \mathbf{f}$ showed the higher molar extinction coefficient than other halogen substituted compounds, and compound $\mathbf{6 e}$ containing $\mathrm{Br}$ substituent exhibited the higher singlet oxygen yield and the lower fluorescence quantum yield than other compounds.
\end{abstract}

Keywords boron diisoindolmethene; synthesis; fluorescence; UV-Vis; singlet oxygen

氟硼二吡咯(Boron dipyrromethene, Bodipy)化合物 是一类重要的荧光染料. 其母体分子结构中两个吡咯环 通过硼桥键和甲川桥键被固定在一个平面上, 形成大 $\pi$ 键共轭，在激发光作用下产生强烈的苂光. Bodipy 染料 通常具有很高的摩尔吸光系数及荧光量子产率, 良好的 光稳定性以及不易受环境、溶液 $\mathrm{pH}$ 的影响等优异的光 化学和光物理性能, 近年来在苂光化学传感器、太阳能 电池感光器、液晶材料、超分子聚合物和光敏剂等领域 有了快速的发展. 典型 Bodipy 染料的紫外吸收和苂光 散射波长在 $530 \mathrm{~nm}$ 左右, 为了得到波长更长的近红外 荧光染料, 通常会在分子结构中引入供电子基团、增加 分子的共轭程度或提高分子的刚性平面或用 $\mathrm{N}$ 原子替
代碳形成氮杂 Bodipy 结构以达到红移的效果 ${ }^{[1 \sim 5]}$, 与此 同时这些化学结构的修饰又往往会影响荧光染料摩尔 吸光系数, 苂光量子产率等其他一些光学性能, 而且这 些结构变化与光学性能之间的相互影响比较复杂. 为了 开发新的苂光染料并了解结构与光学性能的关系, 我们 合成了一系列新的氟硼二异吲哚类化合物 $\mathbf{6 a} \sim \mathbf{6 f}$, 通过 在吡咯环上连接苯环, 达到红移的效果. 同时在结构中 引入卤素取代基, 考察了取代基的变化对其化合物光学 性能的影响. 其中含卤素取代的化合物 $\mathbf{6 c} \sim \mathbf{6 f}$ 为未见报 道的新化合物, 通过对合成的 6 个化合物的紫外可见吸 收光谱、荧光发射光谱的测试发现它们具有较高的摩尔 消光系数、较大的苂光量子产率以及较长的苂光发射波

*E-mail: xiaoxiayang@ecust.edu.cn

Received January 15, 2014; revised February 19, 2014; published online March 13, 2014

Project supported by the National Natural Science Fundation of Chnia (No. 81102314), the Science and Technology Commission of Shanghai Municipality (No. 11ZR1400300) and the Specialized Research Fund for the Doctoral Program of Higher Education (No. 20120074120008).

国家自然科学基金(No. 81102314)、上海市自然科学基金(No. 11ZR1400300)和高等学校博士学科点专项科研基金(No. 20120074120008)资助项目. 
长, 能够作为一类近红外的荧光染料. 卤素取代基对于 化合物的摩尔吸光系数和荧光量子产率有明显的影响. 同时对合成的化合物进行了 1,3-二苯基苯并呋喃 (DPBF) 捕获单线态氧能力的测试, 发现化合物具有一 定的单线态氧的生成能力, 为进一步开发此类化合物作 为光敏剂用于光动力治疗提供参考.

\section{1 结果与讨论}

\section{1 氟嗍二异吲哚类化合物的合成}

以取代的苯甲酸乙酯和邻羟基苯乙酮为原料, 通过 5 步反应合成了化合物 $\mathbf{6 a} \sim \mathbf{6 f}$, 合成路线如 Scheme 1 所 示. 其中化合物 $\mathbf{6 c} \sim \mathbf{6 f}$ 为末见报道的新化合物, 所有化 合物的结构都经 ${ }^{1} \mathrm{H}$ NMR, HRMS, UV 和 IR 的表征.
在 Scheme 1 的合成路线中, 苯甲酸乙酯与水合肼 在乙醇中加热回流高产率( $>90 \%$ )地得到芳基酰肼化合 物 $\mathbf{2} \mathbf{a} \sim \mathbf{2 f}$, 然后与邻羟基苯乙酮反应，脱去一分子水， 得到化合物 3a 3f，产率为 80\% 90\% . 酰腙类化合物 3 生成 1,2-二酰基苯类化合物 4 的反应通常在醋酸碘 苯 ${ }^{[6]}$ 或四醋酸铅 ${ }^{[7]}$ 存在下容易发生，反应机理如 Scheme 2 所示. 在实验中我们分别以化合物 3a, 3d 为原料进行 反应，发现两种氧化剂都能够很好地与酰腙底物反应生 成 1,2-二酰基苯类化合物 $4 \mathbf{a}$ 和 4d, 产率为 50 70\%，其 中四醋酸铅的反应产率(表 1 , Entries 2,6)明显高于醋酸 碘苯(表 1, Entries 1,5). 考虑到四醋酸铅反应过程中用 量较少及后处理方便，最终确定以四醋酸铅为氧化剂来 合成化合物 4 .

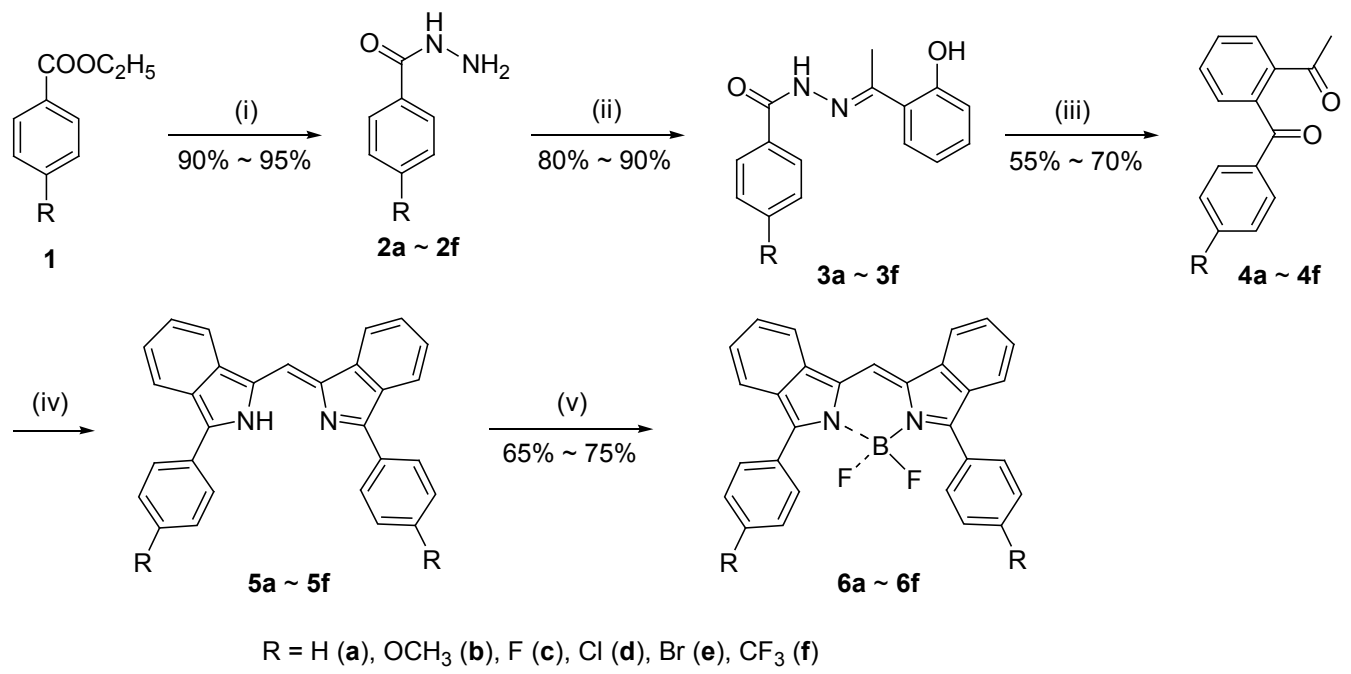

Reagents and conditions: (i) hydrazine hydrate; (ii) 2-acetylphenol; (iii) $\mathrm{Pb}(\mathrm{OAc})_{4}$; (iv) $\mathrm{NH}_{3} \cdot \mathrm{H}_{2} \mathrm{O}, \mathrm{CH}_{3} \mathrm{COOH}, \mathrm{CH}_{3} \mathrm{OH}$; (v) $\mathrm{BF}_{3} \cdot \mathrm{EtO}_{2},(i-\mathrm{Pr})_{2} \mathrm{EtN}, \mathrm{CH}_{2} \mathrm{Cl}_{2}$

Scheme 1

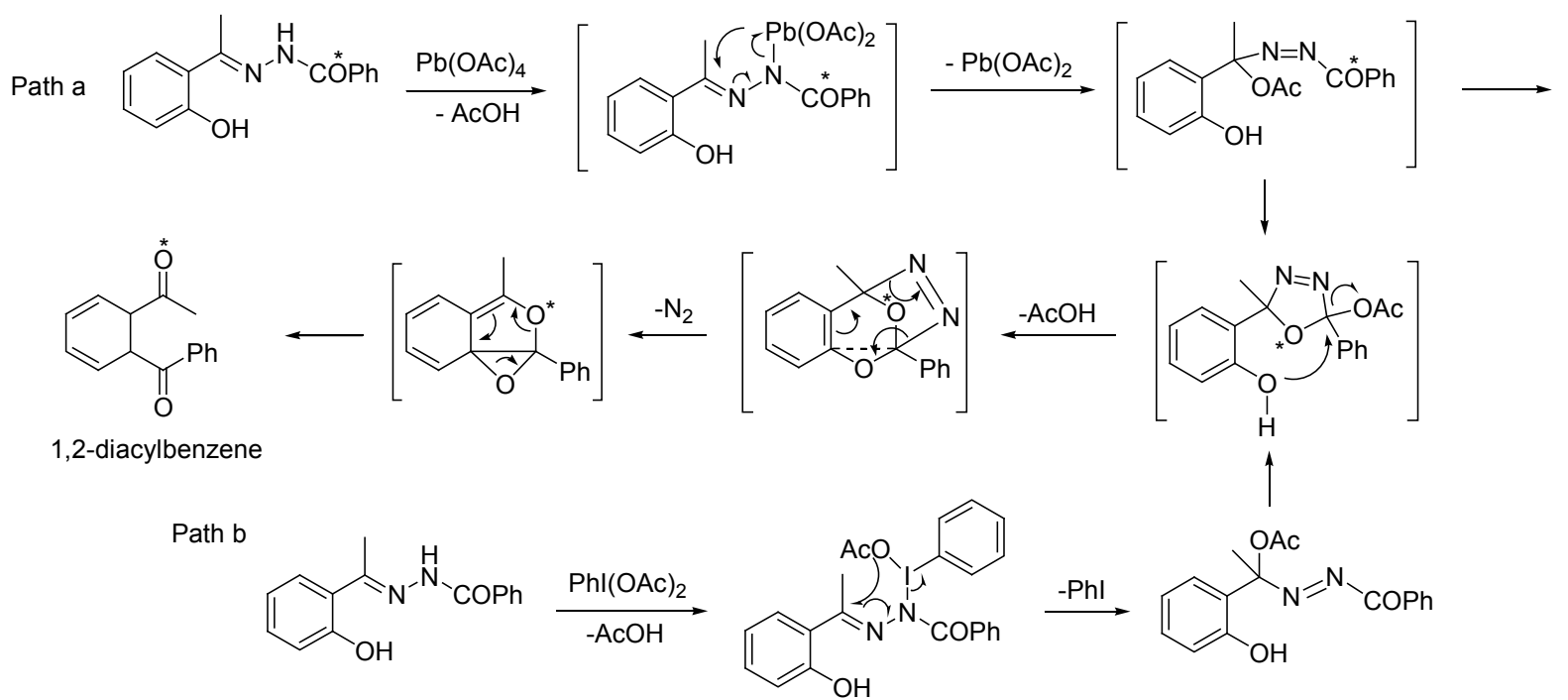

Scheme 2 
表 1 1,2-二酰基苯类化合物的合成 ${ }^{a}$

Table 1 Synthesis of 1,2-diacylbenzenes

\begin{tabular}{ccccc}
\hline Entry & 原料 & 氧化剂 & 产物 & 产率 ${ }^{b} \%$ \\
\hline 1 & 3a & $\mathrm{PhI}(\mathrm{OAc})_{2}$ & $\mathbf{4 a}$ & 60.0 \\
2 & 3a & $\mathrm{Pb}(\mathrm{OAc})_{4}$ & $\mathbf{4 a}$ & 70.4 \\
3 & 3b & $\mathrm{Pb}(\mathrm{OAc})_{4}$ & $\mathbf{4 b}$ & 65.1 \\
4 & 3c & $\mathrm{Pb}(\mathrm{OAc})_{4}$ & $\mathbf{4 c}$ & 60.5 \\
5 & 3d & $\mathrm{PhI}(\mathrm{OAc})_{2}$ & $\mathbf{4 d}$ & 51.7 \\
6 & 3d & $\mathrm{Pb}(\mathrm{OAc})_{4}$ & $\mathbf{4 d}$ & 62.3 \\
7 & 3e & $\mathrm{Pb}(\mathrm{OAc})_{4}$ & $\mathbf{4 e}$ & 55.9 \\
8 & 3f & $\mathrm{Pb}(\mathrm{OAc})_{4}$ & 4f & 55.3 \\
\hline
\end{tabular}

反应条件: $5 \mathrm{mmol}$ 酰腙类化合物, $10 \mathrm{mmol}$ 氧化剂, $70 \mathrm{~mL}$ 溶剂, 室温摚拌; ${ }^{b}$ 分离产率.

二异吲哚亚甲烯化合物 5 是由 2 分子的 1,2-二酰基 苯类化合物 4 在甲酸铵的乙醇溶液中通过脱去一分子甲 醛后生成, 具体反应机理 ${ }^{[8]}$ 见 Scheme 3. 化合物 $\mathbf{4 a}$ 在酸 性条件下与氨作用脱去两分子水生成五元氮杂环中间 体 8, 该中间体不稳定, 环外双键与水发生加成反应生 成异吲哚基甲醇，该化合物在酸性质子脱去一分子水， 形成碳正离子 9 进攻另一份异吲哚基甲醇后, 脱去一分 子甲醛, 再经过空气氧化得到二异吲哚甲烯类化合物 5a. 化合物 $4 b \sim 4 f$ 在铵盐条件都能够顺利的发生此类 反应生成化合物 $\mathbf{5 b} \sim \mathbf{5 f}$ ，但苯环取代基的不同对产率有 明显的影响, 含有供电子基团 $\left(\mathrm{CH}_{3} \mathrm{O}\right)$ 的 1,2-二酰基苯比 含吸电子取代基 $\mathrm{F}, \mathrm{CF}_{3}$ 的化合物更容易发生反应. 实验 中我们也尝试了含硝基取代的 1,2-二酰基苯的反应，但 是并没有得到预期的二异吲哚产物. 推测可能是由于硝
基的强的吸电子作用，不能与氨生成五元杂环的亚胺中 间体 $\mathbf{8}$, 从而使反应不能继续进行.

表 2 二异吲哚甲烯类化合物的合成 ${ }^{a}$

Table 2 Synthesis of bis-isoindolylmethanes

\begin{tabular}{clcc}
\hline Entry & \multicolumn{1}{c}{$\mathrm{R}$} & 产物 & 产率 $^{b} \%$ \\
\hline 1 & $\mathrm{H}$ & $\mathbf{5 a}$ & 67.0 \\
2 & $\mathrm{OCH}_{3}$ & $\mathbf{5 b}$ & 70.3 \\
3 & $\mathrm{~F}$ & $\mathbf{5 c}$ & 52.2 \\
4 & $\mathrm{Cl}$ & $\mathbf{5 d}$ & 55.3 \\
5 & $\mathrm{Br}$ & $\mathbf{5 e}$ & 50.4 \\
6 & $\mathrm{CF}_{3}$ & $\mathbf{5 f}$ & 47.6 \\
7 & $\mathrm{NO}_{2}$ & $-c$ & - \\
\hline
\end{tabular}

反应条件: $5 \mathrm{mmol}$ 1,2-二酰基苯类化合物, $30 \mathrm{~mL}$ 甲醇, $60 \mathrm{mmol}$ 醋酸, 30

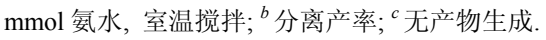

目标化合物 6a 6f 在 Hünig's 碱存在条件下很容易 与 $\mathrm{BF}_{3}$ 的乙醚溶液发生络合，化合物 $\mathbf{5}$ 为蓝紫色粉末状 化合物, 当有目标产物生成时, 溶液颜色变为墨绿色, 通过柱层析可直观的分离得到产物，产率> $75 \%$. 所有 合成的目标产物均通过了 ${ }^{1} \mathrm{H} N \mathrm{~N}$, HRMS 和 IR 的测试.

\section{2 化合物的红外光谱}

对合成化合物 $\mathbf{6 a} \sim \mathbf{6 f}$ 进行了红外光谱的测试，单 环芳烃上的 $=\mathrm{C}-\mathrm{H}$ 的伸缩振动频率在 $3000 \sim 3100$ $\mathrm{cm}^{-1}$, 如果增加共轭链的长度或者变单环为稠环芳烃 $=\mathrm{C}-\mathrm{H}$ 的伸缩振动频率会向高频方向移动. 由表 3 我 们可以看到稠环芳烃上的 $=\mathrm{C}-\mathrm{H}$ 的伸缩振动频率在 $3400 \sim 3460 \mathrm{~cm}^{-1}, \mathrm{C}=\mathrm{C}$ 键的伸缩振动频率在 1600<smiles>CC(=O)c1ccccc1C(=O)c1ccccc1</smiles>

4a<smiles>COc1cccc(O)c1</smiles>
$-\mathrm{H}_{2} \mathrm{O}$

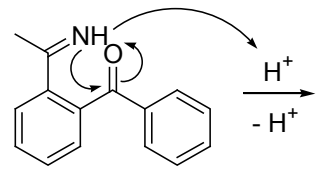<smiles>CC1=NC(O)(c2ccccc2)c2ccccc21</smiles>
7

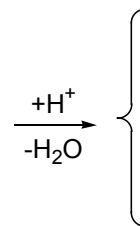

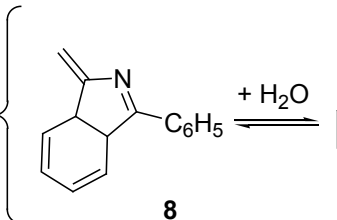

8

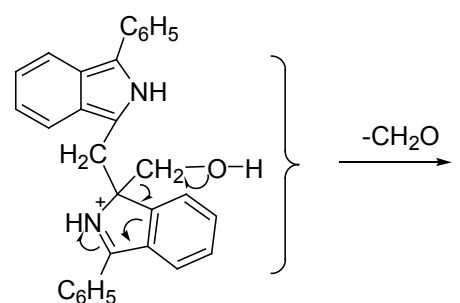

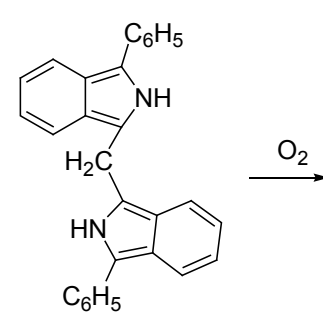

10<smiles>C=C1N=C(CC)c2ccccc21</smiles><smiles>CCCCc1[nH]c(C)c2ccccc12</smiles>

9

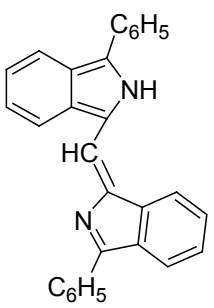

$5 a$<smiles>CCCCCCc1[nH]c(CO)c2ccccc12</smiles>

$\mathrm{C}_{6} \mathrm{H}_{5}$

Scheme 3 
$1620 \mathrm{~cm}^{-1}, \mathrm{C}=\mathrm{N}$ 键的伸缩振动频率在 $1580 \sim 1600$ $\mathrm{cm}^{-1}, \mathrm{~F}-\mathrm{B}$ 键的伸缩振动频率在 $1240 \sim 1270 \mathrm{~cm}^{-1}$. 当 二异吲哚结构中吡咯环上芳环取代基上所连接的基团 是吸电子基时, $\mathrm{C}=\mathrm{N}$ 键的伸缩振动频率逐渐向高频方 向移动; 而 $\mathrm{F}-\mathrm{B}$ 键的伸缩振动频率向低频方向移动(如 化合物 $\mathbf{6 c} \sim \mathbf{6 f}$ ).

表 3 氟硼二异吲哚红外光谱

Table 3 IR spectral data of bis-isoindolylmethanes

\begin{tabular}{ccccc}
\hline 化合物 & $v(=\mathrm{C}-\mathrm{H})$ & $v(\mathrm{C}=\mathrm{C})$ & $v(\mathrm{C}=\mathrm{N})$ & $v(\mathrm{~F}-\mathrm{B})$ \\
\hline $\mathbf{6 a}$ & 3416.6 & 1623.8 & 1591.6 & 1261.3 \\
$\mathbf{6 b}$ & 3422.8 & 1604.2 & 1593.2 & 1261.0 \\
$\mathbf{6 c}$ & 3415.3 & 1618.2 & 1586.7 & 1279.4 \\
$\mathbf{6 d}$ & 3454.5 & 1619.9 & 1590.5 & 1260.3 \\
$\mathbf{6 e}$ & 3409.2 & 1618.8 & 1593.6 & 1243.1 \\
$\mathbf{6 f}$ & 3415.9 & 1613.1 & 1595.2 & 1234.0 \\
\hline
\end{tabular}

\section{3 化合物的紫外吸收光谱}

化合物 $\mathbf{6 a} \sim \mathbf{6 f}$ 在 $\mathrm{CH}_{2} \mathrm{Cl}_{2}$ 溶液中显示了较强的 $\mathrm{S}_{0} \rightarrow \mathrm{S}_{1}$ 的吸收, 最大吸收波长在 $641 \sim 656 \mathrm{~nm}$ (图 1). 相 比较于氟嗍二吡咯母体染料的最大紫外吸收波长 500 $\mathrm{nm}$, 合成的氟硼二异吲哚类化合物, 由于在吡咯结构中 并入了苯环, 增大了分子的共轭范围, 使得化合物的紫 外吸收波长明显红移到 $650 \mathrm{~nm}$ 左右. 苯环上取代基的 变化对于化合物紫外吸收波长的影响也很明显, 如对位 含推电子取代基甲氧基化合物 $\mathbf{6 b}$ 具有最长的紫外吸收 波长 $656 \mathrm{~nm}$, 而 $\mathrm{F}$ 原子取代的化合物 $\mathbf{6 c}$ 和没有取代的 化合物 $6 \mathbf{a}$ 具有相似的吸收波长(表 4, Entries 3 和 1), 含 有吸电子取代基 $\mathrm{Br}$ 和 $\mathrm{CF}_{3}$ 的化合物 $6 \mathbf{e}$ 和 $\mathbf{6 f}$ 的紫外波长 比较于化合物 $6 \mathbf{a}$ 也分别有一定的红移 8 和 $6 \mathrm{~nm}$ (表 4, Entries 5,6). 化合物 $\mathbf{6 a} \sim \mathbf{6 f}$ 普遍具有较强的的摩尔消光 系数 (Table 4), 特别是化合物 $\mathbf{6 b}$ 的摩尔消光系数为 $1.016 \times 10^{5} \mathrm{~L} \cdot \mathrm{mol}^{-1} \cdot \mathrm{cm}^{-1}$, 与酞菁和花菁类染料的摩尔 消光系数相当 $\left(1.5 \sim 2.5 \times 10^{5} \mathrm{~L} \cdot \mathrm{mol}^{-1} \cdot \mathrm{cm}^{-1}\right)$, 含氯原子 取代的化合物 $6 \mathbf{d}$ 显示了相对低的摩尔消光系数 $2.37 \times 10^{4} \mathrm{~L} \cdot \mathrm{mol}^{-1} \cdot \mathrm{cm}^{-1}$. 虽然含 $\mathrm{Br}$ 原子和 $\mathrm{CF}_{3}$ 的化合 物 $6 \mathrm{e}$ 和 $6 \mathbf{f}$ 显示了相似的紫外吸收波长, 但实验发现含 $\mathrm{CF}_{3}$ 取代的化合物 $6 \mathbf{f}$ 具有更强的摩尔消光系数(Table 4, Entry 6).

\section{4 化合物的荧光光谱}

化合物 $\mathbf{6 a} \sim \mathbf{6 f}$ 的苂光光谱测试显示，其荧光发射 波长 $\lambda_{\mathrm{em}}$ 在 $663 \sim 680 \mathrm{~nm}$ (表 5), 苯环上引入推电子甲氧 基时，化合物具有更长的苂光发射波长(680 nm，表 5, Entry 2). 实验所测得的苂光发射光谱与相应的紫外可 见吸收光谱一一对应, 并都有一定的 Stokes 位移, 说明 化合物在光激发过程中, 没有明显的溶剂重组的变化. 化合物 $\mathbf{6 a} \sim \mathbf{6 f}$ 普遍具有较高的荧光量子产率, 但能明

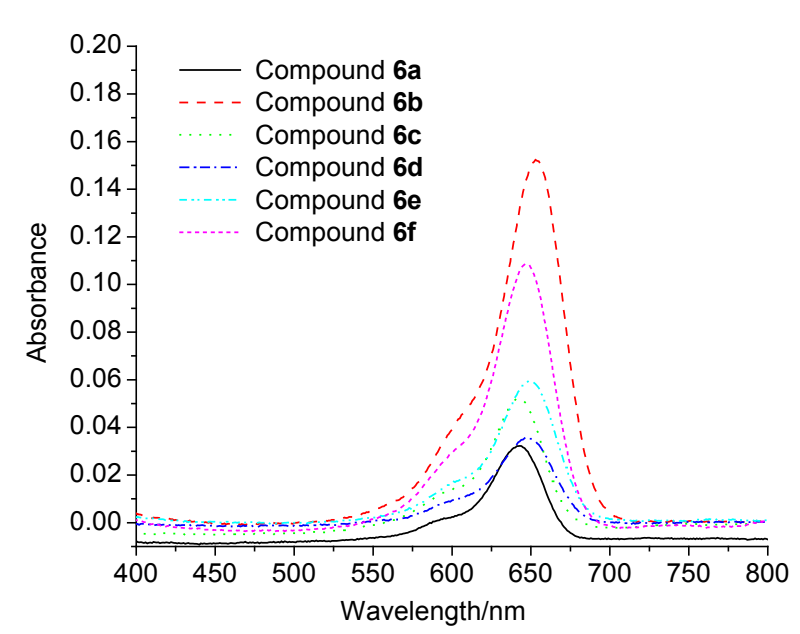

图 1 化合物 $\mathbf{6 a} \sim \mathbf{6 f}$ 在二氯甲烷溶液中的紫外吸收光谱 $(1.5 \times$ $10^{-6} \mathrm{~mol} / \mathrm{L}$, r.t.)

Figure $1 \mathrm{UV}$-visble spectra of compound $\mathbf{6 a} \sim \mathbf{6} \mathbf{f}$ in $\mathrm{CH}_{2} \mathrm{Cl}_{2}$ $\left(1.5 \times 10^{-6} \mathrm{~mol} / \mathrm{L}\right.$, r.t. $)$

表 4 氟嗍二异吲哚 $\mathbf{6 a} \sim \mathbf{6 f}$ 紫外吸收光谱

Table 4 UV-visble spectral data of bis-isoindolylmethanes $6 a \sim 6 f$

\begin{tabular}{|c|c|c|c|c|}
\hline Entry & Compd. & $\mathrm{R}$ & $\lambda_{\max } / \mathrm{nm}$ & $\varepsilon /\left(\mathrm{L}^{\prime} \mathrm{mol}^{-1} \cdot \mathrm{cm}^{-1}\right)$ \\
\hline 1 & $6 a$ & $\mathrm{H}$ & 641 & 21400 \\
\hline 2 & $6 \mathbf{b}$ & $\mathrm{OCH}_{3}$ & 656 & 101600 \\
\hline 3 & $6 c$ & $\mathrm{~F}$ & 643 & 34900 \\
\hline 4 & 6d & $\mathrm{Cl}$ & 646 & 23700 \\
\hline 5 & $6 e$ & $\mathrm{Br}$ & 649 & 39700 \\
\hline 6 & $6 f$ & $\mathrm{CF}_{3}$ & 647 & 72500 \\
\hline
\end{tabular}

显发现，当共轭系统中引入 $\mathrm{Br}$ 原子时，化合物的荧光量 子产率明显下降，化合物 $6 \mathrm{e}$ 的苂光量子产率为 0.64 , 而 分子中含其他取代基如 $\mathrm{F}, \mathrm{Cl}, \mathrm{OCH}_{3}, \mathrm{CF}_{3}$ 时, 对化合物 的苂光量子产率影响不大，这可能是受重原子效应 ${ }^{\left[{ }^{[}\right]}$的 影响，即分子结构中引入重原子基团如 $\mathrm{Br}, \mathrm{I}$ 等取代基 时, 会增大化合物分子从激发态 $\mathrm{S} 1 \rightarrow \mathrm{T} 1$ 的系间交叉转 变的几率, 而降低了化合物分子从 $\mathrm{S}_{1} \rightarrow \mathrm{S}_{0}$ 的转变.

表 5 氟嗍二异吲哚 $\mathbf{6 a} \sim \mathbf{6} \mathbf{6}$ 荧光光谱

Table 5 Fluorescence properties of bis-isoindolylmethanes $6 a \sim 6 f$

\begin{tabular}{cclcc}
\hline Entry & Compd. & \multicolumn{1}{c}{$\mathrm{R}$} & $\lambda_{\text {flu }} / \mathrm{nm}$ & $\Phi_{\mathrm{F}}{ }^{a}$ \\
\hline 1 & $\mathbf{6 a}$ & $\mathrm{H}$ & 663 & $0.73^{b}$ \\
2 & $\mathbf{6 b}$ & $\mathrm{OCH}_{3}$ & 680 & 0.84 \\
3 & $\mathbf{6 c}$ & $\mathrm{F}$ & 663 & 0.85 \\
4 & $\mathbf{6 d}$ & $\mathrm{Cl}$ & 673 & 0.76 \\
5 & $\mathbf{6 e}$ & $\mathrm{Br}$ & 676 & 0.64 \\
6 & $\mathbf{6 f}$ & $\mathrm{CF}_{3}$ & 672 & 0.70 \\
\hline
\end{tabular}

${ }^{a}$ Compound 6a was used as a standard with a literature value for $\Phi_{\mathrm{F}}$ of 0.73 ; ${ }^{b}$ literature value according to Papent US 5433896.

\section{5 单线态氧生成速率的测定}

氟䂽二吡咯类染料作为光敏剂的研究近年来有了 


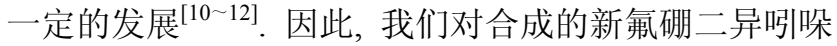
类化合物进行了单线态氧的测定, 以考察这类化合物作 为光敏剂应用. 通过化合物 $\mathbf{6 a} \sim \mathbf{6 f}$ 的 $\mathrm{CH}_{2} \mathrm{Cl}_{2}$ 溶剂在波 长为 $630 \mathrm{~nm}$ 的点状激光照射下, 采用二苯基苯并异呋 喃(DPBF)为探针, 在 $413 \mathrm{~nm}$ 处的紫外吸光度的变化计 算其单线态氧的生成速率, 发现化合物 $\mathbf{6 a} \sim \mathbf{6 f}$ 都有一 定的单线态氧的生成能力. 从紫外光谱图我们发现, 含 DPBF 和化合物 $\mathbf{6 b}$ 的二氯甲烷溶液以及 DPBF 和化合 物 6e 的二氯甲烷溶液 $10 \mathrm{~min}$ 内测得的 $413 \mathrm{~nm}$ 处的吸光 度明显下降(图 2 和图 3), 这说化合物 $\mathbf{6 b}$ 和化合物 $\mathbf{6 e}$ 的单线态氧的生成速率高于其他化合物. 化合物 $6 \mathrm{e}$ 的 这一结果与其荧光光谱中测定的荧光量子产率较低一 致, 正是由于 $\mathrm{Br}$ 原子的引入, 增大了化合物分子从激发 态 $\mathrm{S} 1 \rightarrow \mathrm{T} 1$ 的 intersystem crossing 转变的几率, $\mathrm{T} 1$ 态的分 子容易与周围的 ${ }^{3} \mathrm{O}_{2}$ 发生能量的转化而生成 ${ }^{1} \mathrm{O}_{2}$. 含推 电子甲氧基化合物 $\mathbf{6 b}$ 产生单线态氧的作用机制还有待 进一步研究.

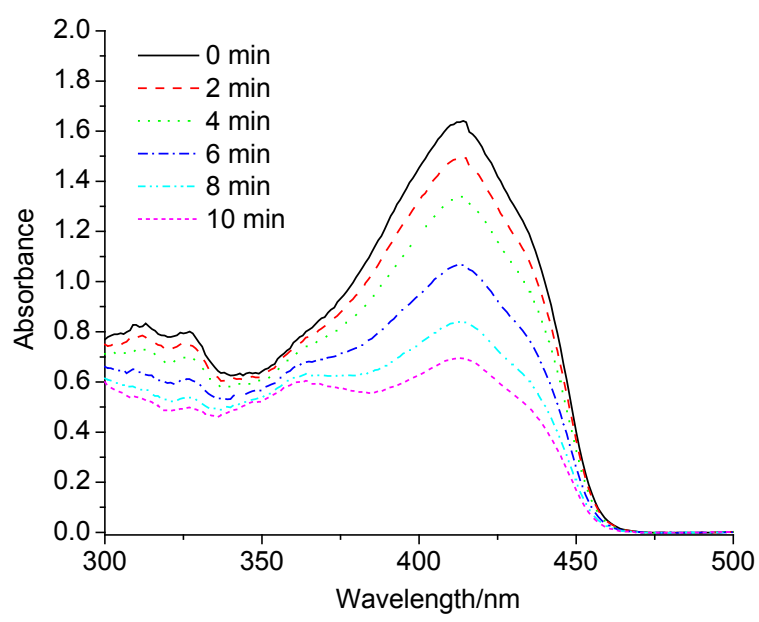

图 2 化合物 $\mathbf{6 b}\left(1.5 \times 10^{-5} \mathrm{~mol} / \mathrm{L}\right)$ 和 DPBF $\left(1.5 \times 10^{-5} \mathrm{~mol} / \mathrm{L}\right)$ 在二氯甲烷中的紫外吸收光谱

Figure 2 UV-vis spectra of compound $6 \mathbf{b}\left(1.5 \times 10^{-5} \mathrm{~mol} / \mathrm{L}\right)$ and DPBF $\left(1.5 \times 10^{-5} \mathrm{~mol} / \mathrm{L}\right)$ in $\mathrm{CH}_{2} \mathrm{Cl}_{2}$

表 6 氟硼二异吲哚 $\mathbf{6 a} \sim \mathbf{6 f}$ 光敏化产生单线态氧的生成速率

Table 6 Ratio of singlet oxygen yield of bis-isoindolylmethanes $\mathbf{6 a} \sim \mathbf{6 f}$ by photosensitivity

\begin{tabular}{ccccccc}
\hline Compd. & 6a & 6b & 6c & 6d & 6e & 6f \\
\hline$k / \mathrm{min}^{-1}$ & 0.017 & 0.086 & 0.031 & 0.037 & 0.063 & 0.023 \\
\hline
\end{tabular}

\section{2 结论}

以取代的苯甲酸乙酯和邻羟基苯乙酮为原料, 通过 5 步合成了一系列氟硼二异吲哚类化合物, 文中详细分 析了合成机理. 其中含卤素取代的化合物 $\mathbf{6 c} \sim \mathbf{6 d}$ 为未 见报道的新化合物, 这些化合物的结构均通过了 ${ }^{1} \mathrm{H}$ NMR，HRMS，IR 等手段的表征. 该系列化合物通过对

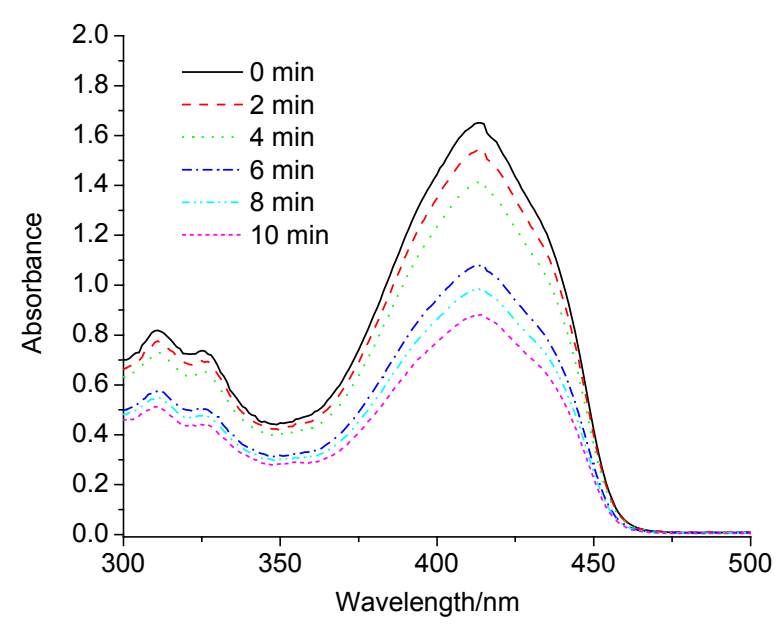

图 3 化合物 $6 \mathrm{e}\left(1.5 \times 10^{-5} \mathrm{~mol} / \mathrm{L}\right)$ 和 DPBF $\left(1.5 \times 10^{-5} \mathrm{~mol} / \mathrm{L}\right)$ 在二氯甲烷中的紫外吸收光谱

Figure 3 UV-visble spectra of compound 6 e $\left(1.5 \times 10^{-5} \mathrm{~mol} / \mathrm{L}\right)$ and DPBF $\left(1.5 \times 10^{-5} \mathrm{~mol} / \mathrm{L}\right)$ in $\mathrm{CH}_{2} \mathrm{Cl}_{2}$

其光谱性能的测试发现, 具有较长的紫外吸收波长 $(640 \sim 650 \mathrm{~nm})$ 和苂光发射波长 $(660 \sim 680 \mathrm{~nm})$, 以及高 的摩尔消光系数及苂光量子产率, 这些优点有利于这类 化合物作为荧光染料进一步开发利用. 同时我们通过 DPBF 捕获单线态氧的实验发现该类化合物具有一定的 单线态氧生成能力, 这些数据可为进一步开发新结构类 型的光敏剂作为参考.

\section{3 实验部分}

\section{1 仪器与试剂}

熔点仪：上海精科仪器有限公司制造 WRS-1B 型; ${ }^{1} \mathrm{H} N M R,{ }^{13} \mathrm{C}$ NMR 为瑞士布鲁克公司 AVANCEIII (400 $\mathrm{MHz}$ )型核磁共振仪, $\mathrm{CDCl}_{3}$ 为溶剂, TMS 为内标; 高分 辨率质谱仪: Bruker Daltonics micrOTOF II; 红外光谱: FT-IR360 型; 紫外光谱仪: Shimadzu UV-2450; 荧光光 谱仪: Cary Eclipse (varian), 激光器: 天津市东港科技发 展有限公司 GY-10 所有试剂均为分析纯，使用之前未经 进一步纯化.

\section{2 实验方法}

\section{2 .1 化合物 $\mathbf{2 a} \sim \mathbf{2 f}$ 的合成}

以苯甲酰肼 $(\mathbf{2 a})$ 的制备为例: 称取苯甲酸乙酯 3.00 $\mathrm{g}(20 \mathrm{mmol}) 、$ 水合肼 $\left(\mathrm{N}_{2} \mathrm{H}_{4} \cdot \mathrm{H}_{2} \mathrm{O}\right) 5.00 \mathrm{~g}(50 \mathrm{mmol})$ 于反应 瓶中, 加入 $50 \mathrm{~mL}$ 无水乙醇摚拌, 加热, 回流 6 小时后, 蒸除大部分乙醇, 有白色的针状晶体析出, 抽滤, 滤饼 干燥，得 $2.51 \mathrm{~g}$ 白色针状晶体 2a, 产率 92.3\%. m.p. $112 \sim 113{ }^{\circ} \mathrm{C}$ (文献值 ${ }^{[13]} 112 \sim 114{ }^{\circ} \mathrm{C}$ ). 采用同样的方法 制备 $\mathbf{2 b} \sim \mathbf{2 f}$.

4- 甲氧基苯甲酰肼 (2b): 白色针状晶体, 产率 
95.1\%. m.p. $134 \sim 136{ }^{\circ} \mathrm{C}$ (文献值 ${ }^{[14]} 136 \sim 138{ }^{\circ} \mathrm{C}$ ).

4-氟苯甲酰肼 (2c): 白色针状晶体, 产率 $90.3 \%$. m.p. $163 \sim 165{ }^{\circ} \mathrm{C}$ (文献值 ${ }^{[14]} 162 \sim 166{ }^{\circ} \mathrm{C}$ ).

4-氯苯甲酰肼(2d): 白色针状晶体, 产率 $89.7 \%$. m.p. $160 \sim 162{ }^{\circ} \mathrm{C}$ (文献值 ${ }^{[15]} 163 \sim 164{ }^{\circ} \mathrm{C}$ ).

4-溴苯甲酰肼(2e): 白色针状晶体, 产率 $90 \%$. m.p. $160 \sim 161{ }^{\circ} \mathrm{C}$ (文献值 ${ }^{[16]} 162 \sim 164{ }^{\circ} \mathrm{C}$ ).

4-三氟甲基苯甲酰肼 (2f): 白色针状晶体, 产率 $87.0 \%$. m.p. $118 \sim 120{ }^{\circ} \mathrm{C}$ (文献值 ${ }^{[17]} 120 \sim 121{ }^{\circ} \mathrm{C}$ ).

\section{2 .2 化合物 $\mathbf{3 a} \sim \mathbf{3 f}$ 的合成}

以 2-[1-(2-羊基苯基)亚乙基]苯甲酰肼(3a)的制备为 例: 称取上步制得的 4-甲氧基苯甲酰肼(2a) $2.49 \mathrm{~g}$ (15 $\mathrm{mmol})$ 和 2-羟基苯乙酮 $2.45 \mathrm{~g}(18 \mathrm{mmol})$ 置于反应瓶中, 加入 $50 \mathrm{~mL}$ 的无水乙醇摚拌, 加热, 回流 $48 \mathrm{~h}$, 待反应 结束后, 有黄色固体析出, 抽滤, 乙醇洗涤 3 次, 干燥, 得 $3.62 \mathrm{~g}$ 黄色针状固体 3a, 产率 85.1\%. m.p. 165 169 ${ }^{\circ} \mathrm{C}\left(\right.$ 文献值 $\left.{ }^{[18]} 164 \sim 165{ }^{\circ} \mathrm{C}\right)$. 采用同样的方法制备 $3 \mathbf{b} \sim$ $3 f$.

2-[1-(2-羊基苯基)亚乙基]-4-甲氧基苯甲酰肼 $(\mathbf{3 b})$ : 黄色针状固体, 产率 $85.1 \%$. m.p. $165 \sim 169{ }^{\circ} \mathrm{C}$.

2-[1-(2-羟基苯基)亚乙基]-4-氟苯甲酰朋(3c): 黄色 针状固体, 产率 $82.2 \%$. m.p. $162 \sim 163{ }^{\circ} \mathrm{C}$.

2-[1-(2-羟基苯基)亚乙基]-4-氯苯甲酰肼(3d): 黄色 针状固体, 产率 $82.8 \%$. m.p. $186 \sim 189{ }^{\circ} \mathrm{C}$.

2-[1-(2-羟基苯基)亚乙基]-4-溴苯甲酰肼(3e): 黄色 针状固体, 产率 $83.0 \%$. m.p. $225{ }^{\circ} \mathrm{C}$.

2-[1-(2-羟基苯基)亚乙基]-4-三氟甲基苯甲酰肼(3f): 黄色针状固体, 产率 $80.7 \%$. m.p. 190 $194{ }^{\circ} \mathrm{C}$.

\section{2 .3 化合物 $\mathbf{4 a} \sim \mathbf{4 f}$ 的合成}

以 1-(2-苯甲酰基苯基)乙酠(4a)的制备为例: 在氩 气的保护下依次加入 $70 \mathrm{~mL}$ 的四氢呋喃, $4.43 \mathrm{~g} \mathrm{(10}$ $\mathrm{mmol}$ )的四醋酸铅, 称取上步制得的 $N$-[1-(2-羊基苯基) 亚乙基]苯甲酰肼(3a) $1.27 \mathrm{~g}(5 \mathrm{mmol}), 0.5 \mathrm{~h}$ 加完, 室温 搅拌 $8 \mathrm{~h}$. 抽滤, 加入少量的四氢呋喃洗涤 3 次, 合并母 液, 蒸出四氢呋喃, 剩余液体用饱和碳酸钠中和到中性, 乙酸乙酯萃取 3 次后蒸除溶剂, 有机相柱层析分离, 洗 脱剂为 $V\left[\right.$ 石油醚 $\left.\left(60{ }^{\circ} \mathrm{C} \sim 90{ }^{\circ} \mathrm{C}\right)\right] ： V($ 乙酸乙酯 $)=10$ : 1 得 $0.74 \mathrm{~g}$ 橙色粉末状固体 $4 \mathrm{a}$, 产率 $65.7 \%$. m.p. 96 98 ${ }^{\circ} \mathrm{C}$; ${ }^{1} \mathrm{H}$ NMR $\left(400 \mathrm{MHz}, \mathrm{CDCl}_{3}\right) \delta: 7.89(\mathrm{~d}, J=7.2 \mathrm{~Hz}$, $1 \mathrm{H}), 7.74(\mathrm{~d}, J=7.2 \mathrm{~Hz}, 2 \mathrm{H}), 7.63 \sim 7.51(\mathrm{~m}, 3 \mathrm{H}), 7.41(\mathrm{t}$, $J=7.6 \mathrm{~Hz}, 3 \mathrm{H}), 2.52(\mathrm{~s}, 3 \mathrm{H})$. 采用同样的方法制备 $\mathbf{4 b} \sim$ $4 \mathbf{4}$.

1-[2-(4-甲氧基苯甲酰基)苯基]乙酮(4b): 橙色粉末 状固体, 产率 65.1\%. m.p. $105 \sim 108{ }^{\circ} \mathrm{C} ;{ }^{1} \mathrm{H}$ NMR (400 $\left.\mathrm{MHz}, \mathrm{CDCl}_{3}\right) \delta: 7.86(\mathrm{~d}, J=7.2 \mathrm{~Hz}, 1 \mathrm{H}), 7.72$ (d, $J=8.8$
$\mathrm{Hz}, 2 \mathrm{H}), 7.64 \sim 7.52(\mathrm{~m}, 2 \mathrm{H}), 7.38(\mathrm{~d}, J=7.6 \mathrm{~Hz}, 1 \mathrm{H})$, $6.90(\mathrm{~d}, J=8.8 \mathrm{~Hz}, 2 \mathrm{H}), 3.84$ (s, 3H), $2.51(\mathrm{~s}, 3 \mathrm{H})$.

1-[2-(4-氟苯甲酰基)苯基]乙酮(4c): 橙色粉末状固 体, 产率 60.5\%. m.p. 137 139 ${ }^{\circ} \mathrm{C} ;{ }^{1} \mathrm{H}$ NMR $(400 \mathrm{MHz}$, $\left.\mathrm{CDCl}_{3}\right) \delta: 7.91 \sim 7.89(\mathrm{~m}, 1 \mathrm{H}), 7.77 \sim 7.73(\mathrm{~m}, 2 \mathrm{H})$, $7.66 \sim 7.59(\mathrm{~m}, 2 \mathrm{H}), 7.39 \sim 7.37(\mathrm{~m}, 1 \mathrm{H}), 7.08(\mathrm{t}, J=8.8$ $\mathrm{Hz}, 2 \mathrm{H}), 2.53$ (s, 3H).

1-[2-(4-氯苯甲酰基)苯基]乙酮(4d): 橙色粉末状固 体, 产率 $61.0 \%$. m.p. $138{ }^{\circ} \mathrm{C} ;{ }^{1} \mathrm{H}$ NMR $(400 \mathrm{MHz}$, $\left.\mathrm{CDCl}_{3}\right) \delta: 7.91(\mathrm{~d}, J=8.0 \mathrm{~Hz}, 1 \mathrm{H}), 7.67 \sim 7.60(\mathrm{~m}, 4 \mathrm{H})$, 7.38 (d, $J=8.4 \mathrm{~Hz}, 3 \mathrm{H}), 2.53$ (s, 3H).

1-[2-(4-溴苯甲酰基)苯基]乙酮(4e): 橙色粉末状固 体, 产率 55.9\%. m.p. $157 \sim 158{ }^{\circ} \mathrm{C} ;{ }^{1} \mathrm{H}$ NMR $(400 \mathrm{MHz}$, $\left.\mathrm{CDCl}_{3}\right) \delta: 7.90(\mathrm{~d}, J=8.0 \mathrm{~Hz}, 1 \mathrm{H}), 7.38(\mathrm{~d}, J=8.4 \mathrm{~Hz}$, $1 \mathrm{H}), 2.55(\mathrm{~s}, 3 \mathrm{H})$.

1-[2-(4-三氟甲基苯甲酰基)苯基]乙酮(4f): 橙色粉 末状固体, 产率 55.3\%. m.p. $105 \sim 108{ }^{\circ} \mathrm{C} ;{ }^{1} \mathrm{H}$ NMR (400 $\left.\mathrm{MHz}, \mathrm{CDCl}_{3}\right) \delta: 7.64(\mathrm{~d}, J=8 \mathrm{~Hz}, 2 \mathrm{H}), 7.56 \sim 7.55(\mathrm{~m}$, $1 \mathrm{H}), 7.40 \sim 7.35$ (m, 2H), 7.03 (d, $J=8.4 \mathrm{~Hz}, 1 \mathrm{H}), 6.96 \sim$ $6.91(\mathrm{~m}, 2 \mathrm{H}), 2.57(\mathrm{~s}, 3 \mathrm{H})$.

\section{2 .4 化合物 $\mathbf{5 a} \sim \mathbf{5 f}$ 的合成}

以 3-苯基-1-[(3-苯基- $2 H$-异吲哚基)亚甲基]- $1 H$-异 吲哚(5a)的制备为例: 称取上步制得的 1-(2-苯甲酰基苯

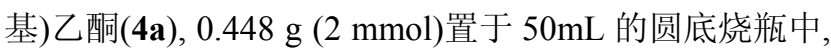
加入 $30 \mathrm{~mL}$ 的甲醇将其溶解, 然后依次向瓶中加入 1.3 $\mathrm{mL}(20 \mathrm{mmol})$ 醋酸和 $0.86 \mathrm{~mL}(10 \mathrm{mmol})$ 的氨水, 室温摚 拌 $4 \mathrm{~h}$. 待反应完毕后, 蒸除甲醇, 饱和碳酸钠溶液将 $\mathrm{pH}$ 值调至中性, 二氯甲烷萃取, 分离出有机相, 干燥后 硅胶进行柱层析分离, 洗脱剂为 $V\left[\right.$ 石油梄 $\left(60{ }^{\circ} \mathrm{C} \sim 90\right.$ $\left.\left.{ }^{\circ} \mathrm{C}\right)\right] ： V($ 乙酸乙酯 $)=10: 1$, 得到 $0.26 \mathrm{~g}$ 深蓝粉末状固 体 5a, 产率 67.0\%. m.p. $235 \sim 237{ }^{\circ} \mathrm{C} ;{ }^{1} \mathrm{H}$ NMR (400 $\left.\mathrm{MHz}, \mathrm{CDCl}_{3}\right) \delta: 8.06 \sim 8.01(\mathrm{~m}, 6 \mathrm{H}), 7.92(\mathrm{~d}, \quad J=8.2 \mathrm{~Hz}$, $2 \mathrm{H}), 7.59 \sim 7.54(\mathrm{~m}, 5 \mathrm{H}), 7.47 \sim 7.43(\mathrm{~m}, 2 \mathrm{H}), 7.38 \sim 7.35$ (m, 2H), 7.32 7.29 (m, 2H). 采用同样的方法制备 $5 \mathbf{b} \sim$ 5f.

3-(4-甲氧基苯基)-1-[[3-(4-甲氧基苯基)- $2 \mathrm{H}$-异吲哚 基]亚甲基]- $1 H$-异吲哚(5b): 深蓝粉末状固体, 产率 70.3\%. ${ }^{1} \mathrm{H}$ NMR $\left(400 \mathrm{MHz}, \mathrm{CDCl}_{3}\right) \delta: 7.89 \sim 7.94(\mathrm{~m}$, $6 \mathrm{H}), 7.85 \sim 7.80(\mathrm{~m}, 3 \mathrm{H}), 7.42(\mathrm{~s}, 1 \mathrm{H}), 7.29 \sim 7.25(\mathrm{~m}$, $3 \mathrm{H}), 7.02 \sim 6.98(\mathrm{~m}, 4 \mathrm{H}), 3.90(\mathrm{~s}, 6 \mathrm{H})$.

3-(4-氟苯基)-1-[[3-(4-氟苯基)- $2 \mathrm{H}$-异吲哚基]亚甲 基]- $1 \mathrm{H}$-异吲哚(5c): 深蓝粉末状固体, 产率 52.2\%. ${ }^{1} \mathrm{H}$ NMR $\left(400 \mathrm{MHz}, \mathrm{CDCl}_{3}\right) \delta: 8.0 \sim 7.90(\mathrm{~m}, 6 \mathrm{H}), 7.62(\mathrm{~d}$, $J=8.8 \mathrm{~Hz}, 2 \mathrm{H}), 7.51 \sim 7.48$ (br s, $2 \mathrm{H}), 7.40 \sim 7.35(\mathrm{~m}$, $3 \mathrm{H}), 7.01 \sim 6.98(\mathrm{~m}, 4 \mathrm{H})$. 
3-(4-氯苯基)-1-[[3-(4-氯苯基)-2 $H$-异吲哚基]亚甲 基]- $1 H$-异吲哚(5d): 深蓝粉末状固体, 产率 $55.3 \%$. ${ }^{1} \mathrm{H}$ NMR $\left(400 \mathrm{MHz}, \mathrm{CDCl}_{3}\right) \delta: 7.90 \sim 8.00(\mathrm{~m}, 7 \mathrm{H}), 7.50 \sim$ $7.58(\mathrm{~m}, 5 \mathrm{H}), 7.38 \sim 7.34(\mathrm{~m}, 2 \mathrm{H}), 7.32 \sim 7.28(\mathrm{~m}, 3 \mathrm{H})$.

3-(4-溴苯基)-1-[[3-(4-溴苯基)-2 $H$-异吲哚基]亚甲 基]- $1 H$-异吲哚(5e): 深蓝粉末状固体, 产率 $50.4 \%$. ${ }^{1} \mathrm{H}$ NMR (400 MHz, $\mathrm{CDCl}_{3}$ ) $\delta: 7.79 \sim 7.71$ (br s, 5H), 7.54 7.48 (m, 8H), 7.13 (d, $J=8.8 \mathrm{~Hz}, 2 \mathrm{H}), 6.81(\mathrm{~d}, J=8.8 \mathrm{~Hz}$, $2 \mathrm{H})$.

3-(4-三氟甲基苯基)-1-[[3-(4-三氟甲基苯基)- $2 H$-异 吲哚基]亚甲基]- $1 H$-异吲哚(5f): 深蓝粉末状固体, 产率 47.6\%. ${ }^{1} \mathrm{H}$ NMR (400 MHz, $\left.\mathrm{CDCl}_{3}\right) \delta: 8.11(\mathrm{~d}, J=8.0 \mathrm{~Hz}$, 2H), $7.98(\mathrm{dd}, J=8.0,2.4 \mathrm{~Hz}, 2 \mathrm{H}), 7.83(\mathrm{~d}, J=8.8 \mathrm{~Hz}$, $2 \mathrm{H}), 7.68 \sim 7.60(\mathrm{~m}, 3 \mathrm{H}), 7.48 \sim 7.32(\mathrm{~m}, 4 \mathrm{H}), 6.90 \sim 7.06$ $(\mathrm{m}, 4 \mathrm{H})$.

\section{2 .5 化合物 $\mathbf{6 a} \sim \mathbf{6 f}$ 的合成}

以二氟 [3- 苯基-1-[(3- 苯基-2 $H$ - 异吲哚基) 亚甲 基]- $1 H$-异吲哚 $-\mathrm{N}_{1}, \mathrm{~N}_{2}$ ]硼(6a)的制备为例: 称取上步制得 的化合物 5a, $0.208 \mathrm{~g}(0.5 \mathrm{mmol})$ 置于 $50 \mathrm{~mL}$ 的圆底烧瓶 中, 加入 $20 \mathrm{~mL}$ 的二氯甲烷将其溶解, 依次向瓶中加入 $0.41 \mathrm{~mL}$ (1.5 mmol)三氟化嗍乙醚溶液和 $0.18 \mathrm{~mL}$ (1 $\mathrm{mmol}$ ) 的 $N, N$-二异丙基乙基胺, 室温搅拌 $7 \mathrm{~h}$. 待反应完 毕后, 溶液变成墨绿色, 蒸除二氯甲烷. 有机物用水洗, 再用二氯甲烷萃取, 无水 $\mathrm{MgSO}_{4}$ 干燥, 分离得有机相, 硅胶柱层析分离, 洗脱剂为 $V\left[\right.$ 石油醚 $\left(60{ }^{\circ} \mathrm{C} \sim 90\right.$ $\left.\left.{ }^{\circ} \mathrm{C}\right)\right]$ : $V($ 乙酸乙酯 $)=10: 1$, 得 $0.162 \mathrm{~g}$ 深绿色针状晶体, 产率 $73.3 \%$. m.p. $275 \sim 276{ }^{\circ} \mathrm{C}$; UV-Vis $\lambda_{\max }: 641 \mathrm{~nm} ;{ }^{1} \mathrm{H}$ NMR (400 MHz, $\left.\mathrm{CDCl}_{3}\right) \delta: 7.91(\mathrm{~d}, J=8.0 \mathrm{~Hz}, 2 \mathrm{H})$, $7.85 \sim 7.81(\mathrm{~m}, 4 \mathrm{H}), 7.62(\mathrm{~d}, J=8.4 \mathrm{~Hz}, 2 \mathrm{H}), 7.51 \sim 7.43$ $(\mathrm{m}, 9 \mathrm{H}), 7.28 \sim 7.21(\mathrm{~m}, 2 \mathrm{H})$. HRESI-MS calcd for $\mathrm{C}_{29} \mathrm{H}_{20} \mathrm{BF}_{2} \mathrm{~N}_{2}[\mathrm{M}+\mathrm{H}]^{+}$445.1609, found 445.1689.

二氟[3-(4-甲氧基苯基)-1-[[3-(4-甲氧基苯基)- $2 H$-异 吲哚基]亚甲基]- $1 H$-异吲哚- $\mathrm{N}_{1}, \mathrm{~N}_{2}$ ] 嗍 $(6 \mathbf{b})$ : 深绿色针状 晶体, 产率 76.4\%. UV-Vis $\lambda_{\max }: 656 \mathrm{~nm} ;{ }^{1} \mathrm{H}$ NMR (400 $\left.\mathrm{MHz}, \mathrm{CDCl}_{3}\right) \delta: 7.86 \sim 7.79(\mathrm{~m}, 4 \mathrm{H}), 7.65 \sim 7.63(\mathrm{~m}, 2 \mathrm{H})$, $7.46 \sim 7.43(\mathrm{~m}, 2 \mathrm{H}), 7.30 \sim 7.11(\mathrm{~m}, 2 \mathrm{H}), 7.03(\mathrm{~d}, J=8.4$ $\mathrm{Hz}, 6 \mathrm{H}), 6.75$ (d, $J=8.4 \mathrm{~Hz}, 2 \mathrm{H}) 3.88$ (s, 6H). HRESI-MS calcd for calcd for $\mathrm{C}_{31} \mathrm{H}_{24} \mathrm{BF}_{2} \mathrm{~N}_{2} \mathrm{O}_{2}[\mathrm{M}+\mathrm{H}]^{+}$505.1821, found 505.1899 .

二氟[3-(4-氟苯基)-1-[[3-(4-氟苯基)-2 $H$-异吲哚基] 亚甲基]- $1 H$-异吲哚- $\left.\mathrm{N}_{1}, \mathrm{~N}_{2}\right]$ 嗍(6c)：深绿色针状晶体，产 率 64.8\%. UV-Vis $\lambda_{\max }$ : $643 \mathrm{~nm} ;{ }^{1} \mathrm{H}$ NMR $(400 \mathrm{MHz}$, $\left.\mathrm{CDCl}_{3}\right) \delta: 7.92(\mathrm{~d}, J=8 \mathrm{~Hz}, 2 \mathrm{H}), 7.85 \sim 7.79(\mathrm{~m}, 5 \mathrm{H}), 7.59$ $(\mathrm{d}, J=8.0 \mathrm{~Hz}, 2 \mathrm{H}), 7.61 \sim 7.44(\mathrm{~m}, 2 \mathrm{H}), 7.29 \sim 7.26(\mathrm{~m}$, 2H), 7.20 (t, $J=8.8 \mathrm{~Hz}, 4 \mathrm{H})$. HRESI-MS calcd for calcd for $\mathrm{C}_{29} \mathrm{H}_{18} \mathrm{BF}_{4} \mathrm{~N}_{2}[\mathrm{M}+\mathrm{H}]^{+}$481.1421, found 481.1489.

二氟[3-(4-氯苯基)-1-[[3-(4-氯苯基)- $2 H$-异吲哚基] 亚甲基]- $1 H$-异吲哚- $\mathrm{N}_{1}, \mathrm{~N}_{2}$ ] 硼(6d)：深绿色针状晶体，产 率 65.8\%. UV-Vis $\lambda_{\max }$ : $646 \mathrm{~nm} ;{ }^{1} \mathrm{H}$ NMR $(400 \mathrm{MHz}$, $\left.\mathrm{CDCl}_{3}\right) \delta: 7.84(\mathrm{~d}, J=8.4 \mathrm{~Hz}, 2 \mathrm{H}), 7.77$ (s, 1H), 7.79 (d, $J$ $=8.4 \mathrm{~Hz}, 4 \mathrm{H}), 7.52(\mathrm{~d}, J=8.0 \mathrm{~Hz}, 2 \mathrm{H}), 7.48 \sim 7.39(\mathrm{~m}$, $6 \mathrm{H}), 7.20 \sim 7.20(\mathrm{~m}, 2 \mathrm{H})$. HRESI-MS calcd for calcd for $\mathrm{C}_{29} \mathrm{H}_{17} \mathrm{BF}_{2} \mathrm{~N}_{2} \mathrm{NaCl}_{2}[\mathrm{M}+\mathrm{Na}]^{+}$535.0830, found 535.0726.

二氟[3-(4-溴苯基)-1-[[3-(4-溴苯基)- $2 H$-异吲哚基] 亚甲基]- $1 H$-异吲哚- $\mathrm{N}_{1}, \mathrm{~N}_{2}$ ]嗍(6e)：深绿色针状晶体，产 率 66.8\%. UV-Vis $\lambda_{\max }$ : $649 \mathrm{~nm} ;{ }^{1} \mathrm{H}$ NMR $(400 \mathrm{MHz}$, $\left.\mathrm{CDCl}_{3}\right) \delta: 7.92(\mathrm{~d}, J=8.4 \mathrm{~Hz}, 2 \mathrm{H}), 7.86(\mathrm{~s}, 1 \mathrm{H}), 7.79$ (d, $J=8.4 \mathrm{~Hz}, 4 \mathrm{H}), 7.60(\mathrm{~m}, 4 \mathrm{H}), 7.51 \sim 7.47(\mathrm{~m}, 4 \mathrm{H}), 7.08 \sim$ $7.05(\mathrm{~m}, 2 \mathrm{H}) ;{ }^{13} \mathrm{C}$ NMR $\left(400 \mathrm{MHz}, \mathrm{CDCl}_{3}\right) \delta: 150.8$, $141.8,134.1,130.6,130.6,130.8,130.9,129.3,128.3$, 124.6, 123.7, 123.3, 123.0, 122.3, 118.0, 114.9 . HRESI-MS calcd for calcd for $\mathrm{C}_{29} \mathrm{H}_{17} \mathrm{BF}_{2} \mathrm{~N}_{2} \mathrm{NaBr}_{2}$ [M+ $\mathrm{Na}]^{+}$622.9820, found 622.9715.

二氟[3-(4-三氟甲基苯基)-1-[[3-(4-三氟甲基苯 基)- $2 H$-异吲哚基]亚甲基]- $1 H$-异吲哚- $\left.\mathrm{N}_{1}, \mathrm{~N}_{2}\right]$ 嗍(6f): 深 绿色针状晶体, 产率 $62.5 \%$. UV-Vis $\lambda_{\text {max }}$ : $647 \mathrm{~nm} ;{ }^{1} \mathrm{H}$ NMR (400 MHz, $\left.\mathrm{CDCl}_{3}\right) \delta: 7.95 \sim 7.90(\mathrm{~m}, 5 \mathrm{H}), 7.77(\mathrm{~d}$, $J=8.4 \mathrm{~Hz}, 4 \mathrm{H}), 7.60(\mathrm{~d}, J=8.4 \mathrm{~Hz}, 2 \mathrm{H}), 7.54 \sim 7.50$ (m, $2 \mathrm{H}), 7.32 \sim 7.29(\mathrm{~m}, 2 \mathrm{H}), 7.04(\mathrm{~d}, J=7.6 \mathrm{~Hz}, 2 \mathrm{H}) ;{ }^{13} \mathrm{C}$ NMR (400 MHz, $\left.\mathrm{CDCl}_{3}\right) \delta: 150.2,134.3,131.4,131.1$, 129.6, 128.2, 125.9, 125.4, 125.3, 123.2, 122.6, 119.1, 115.7. HRESI-MS calcd for calcd for $\mathrm{C}_{31} \mathrm{H}_{18} \mathrm{BF}_{8} \mathrm{~N}_{2}[\mathrm{M}+$ $\mathrm{H}]^{+}$581.1357, found 581.1448.

\subsubsection{Uv-vis 吸收光谱测定}

UV-vis 吸收光谱采用 Shimadzu UV-2450 测定, 记 录波长范围为 $300 \sim 800 \mathrm{~nm}$, 以 $\mathrm{CH}_{2} \mathrm{Cl}_{2}$ 为溶剂, $\mathrm{CH}_{2} \mathrm{Cl}_{2}$ 纯溶剂为基线，高速扫描. 摩尔消光系数的计算根据朗 伯一比尔定律求得. 通过配制不同浓度的溶液, 测定其 吸光度 $A$, 根据吸光度 $A$ 对其浓度 $c$ 作图, 求得线性关 系的斜率即为该吸收波长处的摩尔消光系数.

\subsection{7 荧光光谱测定及荧光量子产率计算}

我们以化合物 $\mathbf{6 a}$ 为标准物, 以化合物 $\mathbf{6 a}$ 的荧光量 子产率 $\left(\Phi_{\mathrm{f}}=0.73\right)^{[19]}$ 作为基准值(Papent US 5433896), $\mathrm{CH}_{2} \mathrm{Cl}_{2}$ 作为溶剂, 配制成稀溶液, 测得荧光发射光谱和 $630 \mathrm{~nm}$ 激发波长下试样的吸光度, 根据公式(1) ${ }^{[20]}$ 求得 化合物的苂光量子产率 $\Phi_{\mathrm{f}}$.

$$
\Phi_{\mathrm{f}}=0.73 \times\left(A_{0} \times S\right) /\left(S_{0} \times A\right)
$$

式中 $\Phi_{\mathrm{f}}$ 为化合物的菼光量子产率, $A_{0}$ 为标准物在 630 $\mathrm{nm}$ 处的吸光度, $S_{0}$ 为基准物荧光发射光谱的积分面积; $A$ 为待测试样在 $630 \mathrm{~nm}$ 处的吸光度, $S$ 为待测试样荧光 


\section{发射光谱的积分面积.}

\section{2 .8 单线态氧生成速率测定}

化合物 $\mathbf{6 a} \sim \mathbf{6 f}$ 光敏化产生单线态氧能力的测定方 法: 以 $\mathrm{CH}_{2} \mathrm{Cl}_{2}$ 为溶剂, 配制含 $15 \mu \mathrm{mol} \cdot \mathrm{L}^{-1}$ 待测试样和 $15 \mu \mathrm{mol} \cdot \mathrm{L}^{-1} \mathrm{DPBF}$ 的混合溶液, 在连续通入氧气的条 件下, 用波长 $630 \mathrm{~nm}$ 的点状激光照射, 采用二苯基苯并 异呋喃为探针, 测定紫外可见吸收光谱在 $413 \mathrm{~nm}$ 处的 吸光度的变化, 根据公式(2)求得单线态氧的生成速率 $k$ 值 ${ }^{[21]}$.

$$
\ln \left(A_{0} / A_{\mathrm{t}}\right)=\mathrm{kt}
$$

式中: $A_{0}$ 为起始浓度下 $413 \mathrm{~nm}$ 处的吸光度, $A_{\mathrm{t}}$ 为某一时 刻 $413 \mathrm{~nm}$ 处的吸光度, $t$ 为激光照射时间, $k$ 为单线态氧 生成速率.

\section{References}

[1] Burghart, A.; Kim, H. J.; Welch, M. B.; Thoresen, L. H.; Reibenspies, J.; Burgess, K.; Bergstrom, F.; Johansson, L. B. A. J. Org. Chem. 1999, 64, 7813.

[2] Yamada, K.; Toyota, T.; Takakura, K.; Ishimaru, M.; Sugawara, T.; New J. Chem. 2001, 25, 667

[3] Atilgan, S.; Ekmekci, Z.; Dogan, A. L.; Guc, D. Akkaya, E. U. Chem. Commun. 2006, 4398

[4] Gorman, A.; Killoran, J.; O'Shea, C.; Kenna, T.; Gallagher, W. M.; O'Shea, D. F. J. Am. Chem. Soc. 2004, 126, 10619
[5] Zhao, W. L.; Carreira, E. M. Chem. Eur. J. 2006, 12, 7254

[6] Moriarty, R. M.; Berghuid, N. A. Synthesis 1993, 318

[7] Katritzky, A. R; Harris, P. A.; Kotali, A. J. Org. Chem. 1991, 56 , 5049

[8] Maekawa, E.; Suzuki, Y.; Sugiyama, S. Chem. Ber. 1968, 101, 847

[9] (a) Yuster, P.; Weissman, S. I. J. Chem. Phys. 1949, 17, 1182. (b) McClure, D. S. J. Chem. Phys. 1949, 17, 905

[10] Lim, S. H.; Thivierge, C.; Nowak-Sliwinska, P.; Han, J. Y. Bergh, H.; Wagnieres, G.; Burgess, K.; Lee, H. B. J. Med. Chem. 2010, 53, 2865.

[11] Awuah, S. G.; You, Y. RSC Adv. 2012, 2, 11169

[12] Kamkaew, A.; Lim, S. H.; Lee, H. B.; Kiew, L. V.; Chung, L. Y.; Burgess, K. Chem. Soc. Rev. 2013, 42, 77

[13] Altalbawy, F. M. A.; Mohamed, G. G.; Mohamed, M. I. A. Asian J. Chem. 2010, 22, 7291 .

[14] Kaushik, D.; Khan, S. A.; Chawla, G. Eur. J. Med. Chem. 2010 45(9), 3960

[15] Saha, A.; Kumar, R.; Kumar, R.; Devakumar, C. Indian J. Chem. 2010, 49B, 526.

[16] Horwitz, J. P.; Grakauskas, V. A. J. Org. Chem. 1954, 19, 194.

[17] Yagupol'skii, L. M.; Gruz, B. E. Ukr. Khem. Zh. 1957, 23, 634.

[18] Kotali, A.; Tsoungas, P. G. Tetrahedron Lett. 1987, 28(37), 4321

[19] Kang, H. C.; Haugland, R. P. US 5433896, 1995 [Chem. Abstr. 1995, 123, 202039].

[20] Umezawa, K.; Matsui, A.; Nakamura, Y.; Citterio, D.; Suzuki, K. Chem. Eur. J. 2008, 15, 1096.

[21] Abimbola, O.; David, M.; Tebello, N. J. J. Mol. Struct. 2003, 650, 131.

(Li, L.; Fan, Y.) 